\section{Conhecimentos e atitudes sobre aleitamento materno em primíparas da cidade do Recife, Pernambuco}

\section{Knowledge and attitudes on breastfeeding among mothers of first-born babies in Recife, Pernambuco}

Ana Maria de Carvalho Albuquerque Melo 1 Poliana Coelho Cabral 2

Elina Albino 3

Lúcia Maria Duque Moura 4

Ana Elizabete Burle de Menezes 5

Luciana Gonçalves Wanderley 6

${ }^{1}$ Laboratório de Nutrição Clínica. Departamento de Nutrição. Centro de Ciências da Saúde. Universidade Federal de Pernambuco. Av. Prof. Moraes Rego, 123. Cidade Universitária. Recife, Pernambuco, Brasil. CEP 50.670-901

2,5 Hospital das Clínicas da Universidade Federal de Pernambuco

${ }^{3}$ Laboratório Central de Saúde Pública da Secretaria de Saúde do Estado de Pernambuco

${ }^{4}$ Hospital da Restauração, Recife, Pernambuco

6 Departamento de Nutrição. Centro de Ciências da Saúde, Universidade Federal de Pernambuco

\section{Resumo}

Objetivos: verificar os conhecimentos das mães sobre amamentação, sua opinião em relação ao uso da mamadeira e chupeta e a participação dos profissionais de saúde na orientação pré e pós-natal sobre aleitamento materno.

Métodos: estudo descritivo de tipo transversal, envolvendo 143 primíparas de 19 maternidades da cidade do Recife, Pernambuco, no período de março de 1997 a outubro de 1998. As mães responderam a um questionário padronizado, contendo informações relativas à amamentação.

Resultados: verificou-se uma elevada prevalência de mães jovens (43\% abaixo dos 19 anos). Apenas 4,2 $\%$ não tinham escolarização e $32,2 \%$ possuíam segundo grau completo ou nível superior. De uma forma geral, $61,6 \%$ das mães tinham pelo menos quatro a sete visitas pré-natais e $51,8 \%$ relataram terem recebido orientação sobre aleitamento durante esse período. Entretanto, com relação às chupetas e mamadeiras, os achados revelaram que respectivamente $80 \%$ e $60 \%$ das mães tinham intenção de comprar e usar esses dois utensílios. Além disso, quase $10 \%$ introduziram chupetas e mamadeiras ainda na maternidade.

Conclusões: este estudo mostra a necessidade de um trabalho multidisciplinar com a finalidade de motivar as mães para promover o aleitamento materno. Isso requer um esforço dos profissionais de saúde no sentido de concientizar as mães que o leite humano é o mais importante alimento para a criança.

Palavras-chave Amamentação, Cuidado do lactente, Alimentação artificial 


\section{Introdução}

O consumo de fórmulas infantis e o uso de mamadeiras e chupetas pelo lactente, fatos que acarretam sérios danos a sua saúde, ainda são freqüentes no Brasil.1

No nosso país, a prática da amamentação está muito aquém da preconizada pela World Health Organization (WHO), ${ }^{2}$ que recomenda o aleitamento exclusivo até os quatro a seis primeiros meses, devendo ser prolongado até o segundo ano de vida do lactente. Em Pernambuco, os resultados da II Pesquisa Estadual de Saúde e Nutrição ${ }^{3}$ revelaram que a taxa de amamentação no total da amostra, no interior urbano e rural, foi de 106, 88 e 109 dias respectivamente, enquanto na região metropolitana do Recife (RMR) foi de 124 dias. Em relação ao aleitamento exclusivo na RMR, $36,4 \%$ das crianças mamaram no primeiro mês decrescendo para $8,4 \%$ no quarto mês e 3,6\% no sexto mês. No interior urbano e rural, os percentuais foram bastante inferiores: $15,1 \%$ e $9,6 \%$ respectivamente no primeiro mês de vida, reduzindo-se para $1,0 \%$ e $0,4 \%$ a partir do quarto mês.

É sabido que nos países do Terceiro Mundo, onde são maiores as desigualdades sociais e desfavoráveis as condições ambientais, a criança de zero a um ano alimentada com fórmulas infantis apresenta risco 14 vezes maior de morrer por diarréia, quando comparada aquela aleitada ao seio. ${ }^{4}$

Alguns estudos têm destacado que o conhecimento das mães sobre amamentação é um dos fatores que contribuem para a adoção dessa prática. 5,6 Jenners, 7 em estudo com primíparas, constatou que a taxa de amamentação exclusiva nos três primeiros meses de vida do bebê, foi significativamente maior entre aquelas que receberam orientação sobre aleitamento materno.

Outro aspecto importante é que o reduzido conhecimento da mãe sobre amamentação implica também em maiores oportunidades para o uso de mamadeiras e chupetas. Esses objetos, quando inadequadamente higienizados, tornam-se veículos de agentes causadores de enteropatias, tais como bactérias, cistos e ovos de parasitos que provocam pro- blemas de má-absorção, anemia e diarréia, levando à desnutrição e muitas vezes, à morte. 8,9

Partindo do pressuposto de que o conhecimento sobre amamentação interfere categoricamente na prevalência e duração dessa prática, os objetivos principais desse estudo foram: 1) verificar os conhecimentos das primíparas participantes do estudo sobre amamentação; 2) conhecer a intenção das primíparas em usar mamadeira e chupeta no período de aleitamento exclusivo e 3) verificar a participação dos profissionais de saúde na orientação pré e pósnatal sobre amamentação.

\section{Métodos}

\section{Desenho do estudo e amostragem}

É um estudo descritivo do tipo transversal realizado com 143 primíparas, atendidas de março de 1997 a outubro de 1998, em 19 das 22 maternidades das redes pública e privada da cidade do Recife, Pernambuco, Brasil, correspondendo a uma cobertura de 90,5\% dos hospitais com este tipo de atendimento.

Considerando o estudo de Victora et al., 1 realizado na cidade de Pelotas no Rio Grande do Sul, no qual $70 \%$ das crianças recém-nascidas fizeram uso de chupeta nos primeiras sete dias de vida, a amostra foi estimada adotando-se um erro de $8 \%$ e um nível de significância de 5\% o que resultou numa amostra mínima de 126 primíparas.

A amostra foi selecionada através de amostragem aleatória sistemática, onde de posse da relação das enfermarias, por pavimento e ala de cada maternidade, procedia-se a escolha da primeira mulher alternando-se sempre uma a partir dela e realizando a entrevista, no caso da mulher ser primípara. Adotando tal critério, ficou determinado como amostra para o estudo, o valor mínimo de $50 \%$ ou mais do número de leitos de cada maternidade (Tabela 1).

É importante salientar que cada primípara selecionada recebia explicações sobre o estudo e só era entrevistada se concordasse em participar. Não houve casos de rejeição em participar da pesquisa. 
Distribuição de mães sorteadas e primíparas entrevistadas segundo maternidade de origem. Recife, Pernambuco, 1997 a 1998.

\begin{tabular}{lcccc}
\hline & Maternidades & Mulheres Internadas & Leitos sorteados & Primíparas \\
\cline { 2 - 4 } Categorias & $(\mathrm{n})$ & $(\mathrm{n})$ & $(\mathrm{n})$ & $(\mathrm{n})$ \\
\hline & & & & \\
Públicas & 10 & 325 & 170 & 83 \\
Particulares & 6 & 152 & 80 & 44 \\
Forças armadas & 2 & 32 & 20 & 9 \\
Filantrópicas & 1 & 36 & 26 & 7 \\
Total & 19 & 545 & 296 & 143 \\
\hline
\end{tabular}

\section{Coleta e processamento dos dados}

O questionário aplicado constou de 34 itens, que abordaram as características pessoais, a história reprodutiva, os conceitos maternos sobre o aleitamento e a atitude da mãe em relação ao uso de mamadeira e chupeta. Todos os questionários foram aplicados pelos autores, no dia seguinte ao parto.

No processamento e análise dos dados, foi utilizado o software Epi-info versão 6.04.10

\section{Resultados}

As características da amostra encontram-se descritas na Tabela 2. Observa-se que $43,4 \%$ das mães tinham menos de 19 anos, sendo classificadas como adolescentes. Por outro lado, apenas $4,2 \%$ das mulheres não sabiam ler nem escrever e 39,2\% tinham nível de instrução igual ou superior ao segundo grau completo.

Quanto à atenção ao pré-natal (Tabela 3), 96,5\% das primíparas tiveram acesso a esse serviço, com um número médio de consultas em torno de $6,4 \pm$ 2,1. Cerca de $46,4 \%$ dessas mulheres iniciaram o pré-natal até o terceiro mês de gestação. No entanto, apesar da elevada freqüência a esse serviço, quase $60 \%$ do total de mães $(n=58)$, relataram não ter recebido nenhum tipo de informação sobre aleitamento materno, e das que receberam $(n=85)$ cerca de $30,6 \%$ a obtiveram já na maternidade (Tabela 4 ).
Quanto às orientações sobre aleitamento dada pelos profissionais dos serviços $52,9 \%$ referiram que a obtiveram através do médico, $21,2 \%$ com enfermeiras e 10,6\% com nutricionistas (Tabela 4).

No que se refere à intenção das mulheres em adquirir e utilizar mamadeiras e chupetas, verifica-se percentuais superiores a $80 \%$ para aquisição desses dois utensílios e $60 \%$ para o seu uso (Tabela 5).

Tabela 2

Distribuição conforme faixa etária e escolaridade de primíparas atendidas em 19 maternidades da cidade do Recife, Pernambuco, 1997 a 1998.

\begin{tabular}{lrr}
\hline Variáveis & $\mathbf{n}$ & $\%$ \\
\hline Faixa etária (anos) & 62 & \\
$<19$ anos & 81 & 43,4 \\
$\geq 19$ anos & 143 & 56,6 \\
$\quad$ Total & & 100,0 \\
Escolaridade & 6 & \\
Nenhuma & 70 & 4,2 \\
$1^{\circ}$ grau completo & 21 & 48,9 \\
$1^{\circ}$ grau completo $+2^{\circ}$ grau incompleto & 46 & 14,7 \\
$2^{\circ}$ grau completo + superior & 143 & 32,2 \\
Total & & 100,0 \\
\hline
\end{tabular}


Tabela 3

Distribuição conforme características do pré-natal de primíparas atendidas em 19 maternidades da cidade do Recife, Pernambuco, 1997 a 1998.

\begin{tabular}{|c|c|c|}
\hline Variáveis & $\mathbf{n}$ & $\%$ \\
\hline \multicolumn{3}{|c|}{ Acesso aos serviços de saúde } \\
\hline Sim & 138 & 96,5 \\
\hline Não & 5 & 3,5 \\
\hline Total & 143 & 100,0 \\
\hline \multicolumn{3}{|l|}{ Número de consultas } \\
\hline $1-3$ & 16 & 11,6 \\
\hline $4-7$ & 85 & 61,6 \\
\hline $8 \mathrm{e}+$ & 37 & 26,8 \\
\hline Total & 143 & 100,0 \\
\hline \multicolumn{3}{|l|}{ Início das consultas } \\
\hline No $1^{\circ}$ trimestre & 64 & 46,4 \\
\hline Após o $1^{\circ}$ trimestre & 74 & 53,6 \\
\hline Total & 143 & 100,0 \\
\hline
\end{tabular}

\section{Tabela 4}

Distribuição conforme orientação sobre aleitamento materno de primíparas atendidas em 19 maternidades da cidade do Recife, Pernambuco, 1997 a 1998.

\begin{tabular}{lrr}
\hline Variáveis & $\mathbf{n}$ & \multicolumn{1}{c}{$\%$} \\
\hline Recebeu orientação & & \\
Sim & 85 & 59,4 \\
Não & 58 & 40,6 \\
Total & 143 & 100,0 \\
Local da orientação & & \\
Serviço de saúde (pré-natal) & 44 & 51,8 \\
Maternidade & 26 & 30,6 \\
Ambos & 15 & 17,6 \\
Total & 85 & 100,0 \\
Quem orientou & & \\
Médico & 45 & 52,9 \\
Enfermeiro & 18 & 21,2 \\
Nutricionista & 9 & 10,6 \\
Agente de saúde & 7 & 8,2 \\
Outros & 6 & 7,1 \\
Total & 85 & 100,0 \\
\hline
\end{tabular}

Tabela 5

Distribuição das primíparas conforme a atitude relativa ao uso de mamadeira ou chupeta atendidas em 19 maternidades da cidade do Recife, Pernambuco, 1997 a 1998.

\begin{tabular}{|c|c|c|c|c|}
\hline \multirow{2}{*}{$\begin{array}{l}\text { Atitudes quanto ao uso de mamadeira ou } \\
\text { chupeta }\end{array}$} & \multicolumn{2}{|c|}{ Mamadeira } & \multicolumn{2}{|c|}{ Chupeta } \\
\hline & $n$ & $\%$ & $\mathrm{n}$ & $\%$ \\
\hline \multicolumn{5}{|l|}{ Comprou / ganhou } \\
\hline Sim & 136 & 95,5 & 126 & 88,1 \\
\hline Não & 7 & 4,9 & 17 & 11,9 \\
\hline Total & 143 & 100,0 & 143 & 100,0 \\
\hline \multicolumn{5}{|l|}{ Uso } \\
\hline Sim & 111 & 77,6 & 87 & 60,8 \\
\hline Não & 28 & 19,6 & 48 & 33,6 \\
\hline Não sabe & 4 & 2,8 & 8 & 5,6 \\
\hline Total & 143 & 100,0 & 143 & 100,0 \\
\hline \multicolumn{5}{|l|}{ Início do uso } \\
\hline Quando chegar em casa & 52 & 46,8 & 52 & 59,8 \\
\hline Depois do $1^{\circ}$ mês & 18 & 16,2 & 5 & 5,8 \\
\hline Depois do $6^{\circ}$ mês & 18 & 16,2 & 5 & 5,8 \\
\hline Quando precisar & 11 & 9,9 & 9 & 10,3 \\
\hline Já está usando & 12 & 10,8 & 7 & 8,0 \\
\hline Total & 111 & 100,0 & 78 & 100,0 \\
\hline
\end{tabular}




\section{Discussão}

$\mathrm{O}$ interesse pelo aleitamento materno ressurgiu na década de 70, a partir de estudos que mostravam aumento da morbimortalidade infantil, em conseqüência ao uso indiscriminado de fórmulas na alimentação nos primeiros meses de vida.11

No entanto, apesar do estímulo a essa prática, segundo dados da II Pesquisa Nacional sobre Saúde e Nutrição, realizada pelo Instituto Nacional de Alimentação e Nutrição (INAN), 12 em 1989, cerca de 43\% das mães abandonavam a amamentação antes do terceiro mês de vida do bebê e apenas $6 \%$ realizavam aleitamento exclusivo até o segundo mês.

Estudos realizados no Brasil sugerem que esse impacto negativo na duração do aleitamento é acarretado pela falta de informação das mães e da sociedade em geral, inclusive dos profissionais de saúde.5-7,11 Sendo assim, é interessante constatar que apesar de muito jovens (43\% adolescentes, faixa etária adequada para assimilação e modificação de condutas) quase $40 \%$ das mulheres estudo tinham nível de instrução igual ou superior ao segundo grau completo, o que reconhecidamente é um fator que contribui para o aprendizado e a motivação para a prática da amamentação.6,9,13

Estudo realizado em Porto Alegre, 5 revelou que mães com um melhor conhecimento sobre aleitamento materno, tinham maior escolaridade e realizaram, no mínimo, cinco consultas pré-natais. Estes resultados são semelhantes aos do presente estudo, no qual além de uma maior escolaridade, as mães tiveram em média cinco consultas pré natais.

Como consequiência a escolaridade materna representa um dos fatores que mais exercem influência na saúde da criança.14,15 Contudo, Susin et al.,6 avaliando o conhecimento de 405 mães de Porto Alegre sobre aleitamento materno, demonstraram que, apesar da escolaridade ter tido importante influência nos conhecimentos das mães, foi possível através de orientação clara e segura, minimizar a desvantagem das mulheres com baixo grau de instrução. No que se refere à frequiência ao pré-natal, os resultados deste estudo são superiores aos encontrados pela II Pesquisa Estadual de Saúde e Nutrição, ${ }^{3}$ onde aproximadamente $85 \%$ das mães de menores de cinco anos referiram essa prática. Porém, enquanto cerca de $2 / 3$ das mulheres, da área urbana ou rural iniciaram o pré-natal no primeiro trimestre de gestação, no nosso estudo esse valor ficou em torno de $46 \%$.

Quanto à orientação sobre o aleitamento, apesar da elevada freqüência ao pré-natal, quase $60 \%$ das mães relataram não terem recebido qualquer tipo de informação, e das que receberam, quase um terço a obteve somente na maternidade. Com base nesses dados, pode-se sugerir que a deficiência dos serviços de saúde com relação a promoção do aleitamento ao seio, pode ser responsável, em grande parte, pelo desmame precoce, pois a escolaridade das mães e a freqüência ao pré-natal não foram empecilhos para uma adequada orientação.

Uma outra questão que merece atenção é saber como essa orientação pode e deve ser realizada bem como decidir qual o profissional mais indicado para assumir tal tarefa. Nesse estudo, das mães que receberam esse tipo de informação, $52,9 \%$ referiram que a obtiveram através do médico e apenas $10,6 \%$ com nutricionistas. Vale salientar a importância do trabalho multidisciplinar nesses casos, no qual cada profissional deve abordar os aspectos mais ligados a sua área, e o mais importante, isso deve ser feito com uma linguagem única e acessível ao grupo de mães a que se destinam as orientações. 16

Essa baixa freqüência de orientação refletiu-se na intenção das mulheres em adquirir e utilizar mamadeiras e chupetas, com percentuais bem superiores a $80 \%$ para aquisição desses dois utensílios e de $60 \%$ para o seu uso. A recomendação de se evitar a chupeta e a mamadeira foi incluída como um dos dez passos para o sucesso da amamentação na Declaração de Innocenti, publicada pelo United Nations Children's Fund (UNICEF), 17 em 1990. A associação entre o uso de chupeta e duração da amamentação foi documentada no início da década de 90 por Victora et al.,18 e tem sido confirmada desde então por outros autores. ${ }^{19}$ Contudo, em 1997, Victora et al.,1 levantaram a questão de que nenhum desses estudos foi desenhado especificamente para testar essa associação, e dados relevantes para essa interpretação poderiam não ter sido coletados.

Vários estudos realizados no Brasil mostram que a frequiência do uso de chupeta foi elevada e a da amamentação foi curta.1,19,20 No presente estudo, $60,8 \%$ e $77,6 \%$ das mães referiram a intenção de uso de chupeta e mamadeira, respectivamente, na chegada em casa. No entanto, dado mais grave, foi o fato de cerca de $10 \%$ das mulheres terem burlado a vigilância das equipes de saúde e introduzido esses utensílios já na maternidade. Conduta que, segundo Victora et al.,15 pode levar à diminuição da frequiência e intensidade da sucção, com conseqüente redução da produção de leite, além do risco de infecções pela contaminação desses utensílios.

Outro fator que merece ser comentado, é que a introdução precoce de outros alimentos através de chucas e mamadeiras, pode interferir na absorção de nutrientes, levando a carências nutricionais. Uma das 
conseqüências dessa prática é o desmame precoce, com a utilização de alimentos inadequados para o lactente, o que pode levar a alergias alimentares e a longo prazo, a doenças como hipertensão, arteriosclerose, obesidade e diabetes mellitus. $21-23$

Em síntese, os dados do presente estudo, são in- dicativos da necessidade de um trabalho de conscientização e esclarecimento junto às mães, que realizado de forma competente e persistente, por uma equipe de pré-natal multidisciplinar, levará as mães a ter maior consciência da importância do aleitamento materno para a saúde de seus filhos.

\section{Referências}

1. Victora CG, Behague DP, Barros FC, Olinto MTA, Weiderpass E. Pacifier use and short breastfeeding duration: cause, consequence, or coincidence? Pediatrics 1997; 99: 445-53.

2. WHO (World Health Organization). Infant-feeding recommendations. Bull World Health Organ 1995; 73: 165-74.

3. Pernambuco. Secretaria de Saúde. II Pesquisa Estadual de Saúde e Nutrição: Pernambuco. Recife: Secretaria de Estado de Saúde; 1997.

4. Victora CG, Smith PG, Vaughan JP. Evidence for a strong protective effect of breast-feeding against infant deaths due to infectious disease in Brazil. Lancet 1987; 2: 31922 .

5. Giugliani ERJ, Rocha VLL, Neves JM, Polanczyk CA, Seffrin CF, Susin LRO. Conhecimentos maternos em amamentação e fatores associados. J Pediatr (Rio de Janeiro): 1995; 71: 77-81.

6. Susin LRO, Giugliani ERJ, Kummer SC, Maciel M, Benjamin ACW, Machado DB, Barcaro M, Draghetti V. Uma estratégia simples que aumenta os conhecimentos das mães em aleitamento materno e melhora as taxas de amamentação. J Pediatr (Rio de Janeiro) 1998; 74: 368-75.

7. Jenners $S$. The influence of additional information, advice and support on the sucess of breastfeeding in working class primiparas. Child Care Health Dev 1988; 14: 31928.

8. Pedroso RS, Siqueira RV. Pesquisa de cistos de protozoários, larvas e ovos de helmintos em chupetas. J Pediatr (Rio de Janeiro) 1997; 73: 21-5.

9. Primo CC, Caetano LC. A decisão de amamentar da nutriz: percepção de sua mãe. J Pediatr (Rio de Janeiro) 1999; 75: 449-55

10. WHO (World Health Organization). Epi-Info: a word processing, database and statistic program for public health: version 6.04b. Atlanta: Center of Disease Control \& Prevention; 1997.

11. Lamounier JA, Leão, E. Nutrição na infância. In: Dutra-deOliveira JE, Marchini JS. Ciências nutricionais. São Paulo: Sarvier; 1998. p. 218-37

12. INAN (Instituto Nacional de Alimentação e Nutrição). Pesquisa Nacional sobre Saúde e Nutrição: perfil de

Recebido em 2 de outubro de 2001

Versão final representada em 2 de julho de 2002

Aprovado em 14 de julho de 2002

crescimento da população brasileira de 0 a 25 anos. Brasília, DF: Ministério da Saúde; 1990.

13. Jacobson SW, Jacobson JL, Frye KF. Incidence and correlates of breast-feeding in socioeconomically disadvantaged women. Pediatrics 1991; 88: 728-36.

14. Cleland JG, van Ginneken JK. Maternal education and child survival in developing countries: the search for pathways of influence. Soc Sci Med 1988; 27: 1357-68.

15. Victora CG, Huttly SRA, Barros FC, Lombardi C, Vaughan JP. Maternal education in relation to early and late child health outcomes: findings from a Brazilian cohort study. Soc Sci Med 1992; 34: 899-905.

16. Grossman LK, Harter C, Hasbrouck C. Testing mother`s knowledge of breastfeeding: instrument development and implementation and correlation with infant feeding decision. J Perinat Nutr 1990; 2: 43-63.

17.UNICEF (United Nations Children's Fund), WHO (World Health Organization). Innocenti declaration on the protection, promotion and support of breastfeeding. Florence: UNICEF, WHO; 1990

18. Victora CG, Tomasi E, Olinto MTA, Barros FC. Use of pacifiers and breastfeeding duration. Lancet 1993; 341 : 404-6.

19. Barros FC, Victora CG, Semer TC, Toniolo Filho S, Tomasi E, Weiderpass E. Use of pacifiers is associated with decreased breastfeeding duration. Pediatrics 1995; 95: 497 -

20. Carvalho M. Obstáculos ao aleitamento materno: fatos e mitos. J Pediatr (Rio de Janeiro) 1985; 50: 403-14.

21. Newman V. Position of the American Dietetic Association: promotion and support of breast-feeding. J Am Diet Assoc 1993; 43: 467-9.

22. Ravelli ACJ, Vander Meulen JHP, Osmand C, Barker DJP, Bleker OP. Infant feeding and adult glucose tolerance, lipid profile, blood pressure and obesity. Arch Dis Child 2000; 82: 248-52.

23. Spolidoro JVN, Müller DP. Alimentação no $1^{\circ}$ ano de vida Rev Bras Nutr Clin 2001; 16: 175-9. 